\title{
Erratum to: Post-relapse survival in patients with the early and late distant recurrence in estrogen receptor-positive HER2-negative breast cancer
}

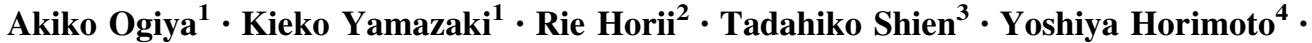 \\ Norikazu Masuda $^{5}$. Touko Inao ${ }^{6}$. Mitsuchika Hosoda ${ }^{7} \cdot$ Naoko Ishida $^{7}$. \\ Tomofumi Osako $^{8,9} \cdot$ Masato Takahashi $^{10}$ - Yumi Endo ${ }^{11}$ - Yuichiro Miyoshi ${ }^{3}$. \\ Hiroyuki Yasojima ${ }^{5}$ Nobumoto Tomioka ${ }^{10}$ - Hiroko Yamashita ${ }^{7}$. \\ Collaborative Study Group of Scientific Research of the Japanese Breast Cancer Society
}

Published online: 4 April 2017

(c) The Japanese Breast Cancer Society 2017

\section{Erratum to: Breast Cancer \\ DOI 10.1007/s12282-016-0730-3}

Unfortunately, in the original publication of this article, the $P$ value of "ER (\%), median (range)" was misplaced to previous row "Tumor grade 3" in Table 4.

The original article was corrected.

The online version of the original article can be found under doi:10.1007/s12282-016-0730-3.

Hiroko Yamashita

hirokoy@huhp.hokudai.ac.jp

1 Breast Surgical Oncology, Cancer Institute Hospital, Japanese Foundation for Cancer Research, Tokyo, Japan

2 Division of Pathology, Cancer Institute of the Japanese Foundation for Cancer Research, Tokyo, Japan

3 Breast and Endocrine Surgery, Okayama University Hospital, Okayama, Japan

4 Department of Breast Oncology, Juntendo University School of Medicine, Tokyo, Japan

5 Department of Surgery, Breast Oncology, NHO Osaka National Hospital, Osaka, Japan

6 Department of Breast and Endocrine Surgery, Graduate School of Medical Science Kumamoto University, Kumamoto, Japan
Breast Surgery, Hokkaido University Hospital, Kita 14, Nishi 5, Kita-ku, Sapporo 060-8648, Japan

8 Department of Breast and Endocrine Surgery, Kumamoto City Hospital, Kumamoto, Japan

9 Present Address: Kumamoto Shinto General Hospital, Kumamoto, Japan

10 Department of Breast Surgery, NHO Hokkaido Cancer Center, Sapporo, Japan

11 Department of Oncology, Immunology and Surgery, Nagoya City University Graduate School of Medical Sciences, Nagoya, Japan 